\title{
Consumo de tabaco en población menor de 18 años: estudio de prevalencia en escolares de Chile
}

\author{
Gonzalo Valdivia ${ }^{1}$ Franco Simonettia , Patricio Cumsillea, \\ Valeria Ramíreza, Carmen G loria Hidalgoa, \\ Beatriz Palmaa, Juan Carrascob. \\ Smoking habit in school age \\ children, in Chile
}

\begin{abstract}
Background: In Chile, there is no information about the prevalence of smoking among basic and high school students. Aim: To study the prevalence of smoking among school age population. Material and Methods: A cross sectional survey was conducted during 2001, assessing tobacco use and smoking habit in a representative sample of 15.119 randomly selected students. Results: Sixty four percent of students had smoked at least once in their lifetime. Eighteen percent of students smoked daily and $15 \%$, occasionally. The figures for women were higher (19 and 18\% respectively). The mean age for the first contact with smoking was 12.3 years and, for starting the smoking habit, 13.2 years. Smokers increased the amount of cigarettes during weekends. Higher prevalence rates of smoking were observed in students from the Southern region of the country and of lower socioeconomic levels. From the 3rd grade on, there is an increased risk of having contact with tobacco and smoking for the first time and from 6th grade on, this risk becomes permanent. The adjusted Odds Ratio to be a smoker is significantly higher when the mother is a current or irregular smoker (OR 1.9 $95 \mathrm{CI}$; 1.7-2.0). Conclusions: High smoking prevalence rates were detected in this survey, mainly in women. The risk for smoking starts early during school life. Therefore, health promotion programs must include elementary and high school students (Rev Méd Chile 2004; 132: 171-82).
\end{abstract}

(Key Words: Adolescent behavior; Data collection; Smoking)

Recibido el 16 de julio, 2003. Aceptado en versión corregida el 26 de noviembre, 2003.

${ }^{1}$ Departamento de Salud Pública, Facultad de Medicina, Escuela de Psicología, Pontificia Universidad Católica de Chile. Santiago, Chile.

a Psicólogo

${ }^{b}$ Estudiante de Medicina, Pontificia Universidad Católica de Chile.

$\mathrm{C}$ hile se encuentra en una situación de transición epidemiológica avanzada y en su población se ha constatado una alta frecuencia de

Correspondencia a: Dr. Gonzalo Valdivia Cabrera. Departamento de Salud Pública, Facultad de Medicina, Pontificia Universidad Católica de Chile. Marcoleta 352, Santiago Chile. Fax: 6331840. E mail: valdivia@med.puc.cl factores de riesgo de enfermedad crónica ${ }^{1-3}$. Las intervenciones para modificar el perfil de algunos de estos factores, como ser el consumo de alcohol y tabaco, requieren disponer de información actualizada sobre la prevalencia de ellos en la población. Chile cuenta con adecuada información epidemiológica en población adulta, especialmente en cuanto a consumo de tabaco ${ }^{2-8}$. Sin 
embargo, en población infantil escolar se precisa mayor información a fin de conocer mejor la historia natural del consumo de tabaco en este grupo. Estudios nacionales han abordado parcialmente el problema, predominando aquellos centrados en escolares de sectores urbanos y que excluyen a niños del ciclo básico ${ }^{9-18}$. La intervención en prevención primaria sobre el tabaquismo debe iniciarse precozmente en la vida, siendo relevante para esto conocer los patrones de consumo y la influencia de variables conductuales que participan en el consumo de tabaco en escolares $^{19-22}$. Es de interés conocer la prevalencia del tabaquismo en escolares por su importancia futura respecto de numerosas patologías y por la influencia del hábito en las capacidades de aprendizaje del escolar. El tabaquismo se ha vinculado también con el consumo de otras sustancias, como drogas ilícitas y alcohol y con el desarrollo de algunas conductas poco deseables en el niño y adolescente ${ }^{23}$.

Con la finalidad de obtener información nacional sobre la prevalencia y características del consumo de tabaco en escolares chilenos, llevamos a cabo un estudio de prevalencia en una muestra nacional representativa del sistema escolar chileno de enseñanza básica y media. Se investigó también la influencia de variables psicosociales y epidemiológicas en la prevalencia de consumo de tabaco en niños y adolescentes ${ }^{24,25}$.

\section{Materiales y MÉTOdo}

Se llevó a cabo un estudio de prevalencia durante el primer semestre del año 2001. La población estudiada fueron escolares, cursando entre el $1^{\circ}$ de enseñanza básica y $4^{\circ}$ año de enseñanza media, de colegios particulares pagados, particulares subvencionados, y corporaciones municipales (3.149.000 alumnos en el año 2000). Se efectuó un muestreo probabilístico que consideró: a) selección al azar de regiones en tres zonas geográficas; b) selección al azar de Departamentos Provinciales de Educación en las regiones seleccionadas; c) selección al azar de comunas en éstos; d) selección al azar de establecimientos; y e) selección al azar de cursos. La distribución geográfica de las zonas fue: zona norte, comprendiendo desde la I a la IV regiones (seleccionando las regiones II y IV), zona central, compren- diendo desde la $\mathrm{V}$ a la VI regiones (seleccionando las regiones $\mathrm{V}$ y metropolitana) y zona sur, comprendiendo desde la VII a la XII regiones (seleccionando las regiones VII y VIII). Se evaluó a los alumnos asistentes el día de aplicación de los instrumentos. En el caso de niños de $1^{0}$ a $3^{\circ}$ básico, se seleccionó al azar a 5 niños de cada curso. Para el cálculo del tamaño muestral se estimó $2 \%$ de consumo de tabaco en niños entre 6-10 años, 14\% entre los de 11-13 años y 40\% en los de 14 a 17 años, asumiendo una mayor prevalencia de consumo en varones del primer grupo de edad y mayor consumo en mujeres en los grupos restantes. Se supuso que no habría diferencia de consumo según nivel socioeconómico (NSE) y zona geográfica, asumiendo un efecto de diseño de 1,5 . Con $2 \%$ de error muestral, el tamaño muestral calculado fue de 16.356 escolares, estimándose que las submuestras debían ser de 846 en el grupo de $1^{0}$ a $5^{0}$ básico, 5.190 en el de $6^{0}$ a $8^{\circ}$ básico y 10.320 en el grupo de $1^{\circ}$ a $4^{\circ}$ medio, manteniendo igual proporción de niños según nivel socioeconómico, zona geográfica y sexo. Los errores muestrales fluctuaron entre 1,35\% (menores de 11 años NSE alto) y 4,65\% (escolares mayores de 14 años, zona sur). Como estimador del NSE se usó el nivel de dependencia del establecimiento (colegios particulares pagados como indicador de NSE alto, particulares subvencionados y corporaciones como de NSE medio y los colegios municipales como indicador de NSE bajo).

Se utilizaron las siguientes variables relacionadas con el consumo de tabaco: prueba de cigarrillos (sujeto probador), fumador diario (al menos 1 cigarrillo/día), fumador ocasional (menos de un cigarrillo/día), fumador explícito (diarios u ocasionales) y sujetos ex-fumadores (sujeto probador, fumador posterior y que se mantiene sin consumo al menos un mes previo a la entrevista). Se incluyeron también variables como edad de inicio y frecuencia de consumo, actitud frente al tabaquismo, conocimiento de efectos para la salud, conducta de abandono actual y perspectiva futura de consumo. Se evaluaron también variables que influyeron en el inicio del consumo de tabaco, el modelo de consumo de tabaco en padres, amigos o compañeros, fuente de obtención de cigarrillos y disponibilidad de éstos entre pares y familiares.

Instrumentos. Se utilizó un cuestionario elaborado especialmente para esta investigación, incor- 
porando algunas preguntas utilizadas en investigaciones similares realizadas tanto en Chile como en otros países $24,26,27$. El instrumento fue evaluado y modificado posterior a una aplicación piloto, siendo autoadministrado en escolares de 50 año básico o cursos superiores, y administrado por un entrevistador a escolares de $1^{\underline{0}}$ a $4^{\underline{0}}$ básico. Se elaboró un manual de administración y hojas de respuesta para lectura óptica de datos. Coordinadores locales fueron capacitados en la aplicación del instrumento por el equipo investigador, aplicando el instrumento entre el 22 de mayo y el 4 de junio de 2001. El análisis se efectuó utilizando el programa Stata 7.0, calculando prevalencias simples según algunas variables epidemiológicas de interés. Odds Ratio (OR) de prevalencias crudos y ajustados fueron también calculados con sus correspondientes intervalos de confianza (IC: 95\%), ajustando sus valores por un conjunto de potenciales variables confundentes. El estudio contó con el consentimiento de las autoridades locales y de los padres 0 apoderados.

\section{RESULTADOS}

Se obtuvo información válida de 17.297 escolares, cifra que se reduce a 16.383 niños con registros validados respecto de las principales variables y covariables de interés para el estudio (Tabla 1). Para los tres grupos de edad se obtuvieron sub-

\section{Tabla 1. D istribución muestral de escolares según grupo de edad, nivel socioeconómico estimado, zona del país y sexo de los alumnos entrevistados}

\begin{tabular}{|c|c|c|c|c|}
\hline \multicolumn{5}{|c|}{ Sexo } \\
\hline Grupos de edad $^{(1)}$ & $\begin{array}{l}\text { Masculino } \\
\text { n (\%) }\end{array}$ & \multicolumn{2}{|c|}{$\begin{array}{l}\text { Femenino } \\
\mathrm{n}(\%)\end{array}$} & $\begin{array}{l}\text { Total } \\
\text { n (\%) }\end{array}$ \\
\hline 6-10 años & $440(5,5)$ & \multicolumn{2}{|c|}{$425(5,1)$} & $865(5,3)$ \\
\hline 11-14 años & $2.525(31,4)$ & \multicolumn{2}{|c|}{$2.574(30,9)$} & $5.099(31,1)$ \\
\hline Más de 14 años & $5.076(63,1)$ & \multicolumn{2}{|c|}{$5.343(64,05)$} & $10.419(63,6)$ \\
\hline \multirow[t]{3}{*}{ Total } & $8.041(100)$ & \multicolumn{2}{|c|}{$8.342(100)$} & $16.383(100)$ \\
\hline & \multicolumn{4}{|c|}{ Nivel socio económico(2) } \\
\hline & $\begin{array}{l}\text { Alto } \\
\text { n (\%) }\end{array}$ & $\begin{array}{l}\text { Medio } \\
\mathrm{n}(\%)\end{array}$ & $\begin{array}{l}\text { Bajo } \\
\text { n (\%) }\end{array}$ & $\begin{array}{l}\text { Total } \\
\text { n (\%) }\end{array}$ \\
\hline 6-10 años & $289(5,3)$ & $307(5,6)$ & $269(5,0)$ & $865(5,3)$ \\
\hline 11-14 años & $1.772(32,4)$ & $1.761(31,8)$ & $1.566(29,1)$ & $5.099(31,1)$ \\
\hline Más de 14 años & $3.416(62,3)$ & $3.456(62,6)$ & $3.547(65,9)$ & $10.419(63,6)$ \\
\hline \multirow[t]{3}{*}{ Total } & $5.477(100)$ & $5.524(100)$ & $5.382(100)$ & $16.383(100)$ \\
\hline & \multicolumn{4}{|c|}{ Zona geográfica de vivienda(3) } \\
\hline & $\begin{array}{l}\text { Norte } \\
\text { n (\%) }\end{array}$ & $\begin{array}{l}\text { Centro } \\
\text { n (\%) }\end{array}$ & $\begin{array}{l}\text { Sur } \\
\mathrm{n}(\%)\end{array}$ & $\begin{array}{l}\text { Total } \\
\text { n (\%) }\end{array}$ \\
\hline 6-10 años & $307(5,6)$ & $280(5,1)$ & $278(5,2)$ & $865(5,3)$ \\
\hline 11-14 años & $1.698(31,0)$ & $1.674(30,2)$ & $1.727(32,2)$ & $5.099(31,1)$ \\
\hline Más de 14 años & $3.474(63,4)$ & $3.583(64,7)$ & $3.362(62,6)$ & $10.419(63,6)$ \\
\hline Total & $5.479(100)$ & $5.537(100)$ & $5.367(100)$ & $16.383(100)$ \\
\hline
\end{tabular}

${ }^{(1)}$ Grupos de edad equivalentes a: 1ํa 50 año Básico, 60 a $8^{0}$ año básico y $1^{10}$ a $4^{0}$ año medio respectivamente. ${ }^{(2)}$ Nivel socioeconómico estimado según dependencia del establecimiento: alto (establecimientos particulares pagados), medio (establecimientos particulares subvencionados), bajo (establecimientos fiscales). (3)Zona norte: $1^{\underline{a}}$ a $4^{\underline{a}}$ región; zona central: regiones $5 \stackrel{a}{\underline{a}}$, metropolitana y $6 \underline{a}$; zona sur: regiones $7 \underline{a}$ a $12 \stackrel{a}{\text { a }}$. 
muestras de tamaños adecuados de acuerdo a la estimación previa. La distribución por sexo fue de $49,1 \%$ varones (8.041) y 50,9\% mujeres (8.342). La distribución según nivel socioeconómico estimado (NSE) fue de 33,4\% (5.477) para el NSE alto, 33,7\% (5.524) para el NSE medio y 32,9\% (5.382) para el NSE bajo. Según zona geográfica, la zona norte correspondió 33,4\% (5.479), la zona central 33,8\% (5.537) y la zona sur 32,8\% (5.367) (Tabla 1).

Prevalencia del consumo de tabaco. Luego del uso de algoritmos de consistencia de respuestas, se obtuvo un total de 15.119 observaciones válidas para el análisis. El 64,1\% (95\% IC: 63,3-64,8) de los niños y jóvenes refirieron haber probado 0 fumado cigarrillos en su vida. La frecuencia fue de $67,7 \%$ para mujeres (95\% IC: $66,6-68,7)$ y de $60,4 \%$ para varones (95\% IC: 59,2-61,5) ( $p<0,0001)$. Esta diferencia en el consumo por sexo se acentuó a partir de 70 año básico, alcanzando a 73\% de tabaquismo explícito en mujeres cursando $4^{0}$ año de enseñanza media.

En ambos sexos, las prevalencias de tabaquismo explícito aumentaron conforme mayor era la edad de los alumnos encuestados, con tasas siempre mayores para las mujeres. También el nivel socioeconómico bajo presentó las mayores tasas de prevalencia, lo que fue especialmente notorio a partir de los 11 años de edad. De igual manera, los escolares entrevistados en la zona sur del país tuvieron las menores tasas de tabaquismo explícito (Tablas 2 y 3 ).

Los varones tuvieron mayor frecuencia de no fumadores que las mujeres $(39,4 \%$ y $32,1 \%$ : p $<0,0001$ ) (Tabla 2). Las mujeres tuvieron también mayores tasas de tabaquismo diario que varones $(19,2 \%$ y $16,3 \%$; p <0,0001) y de tabaquismo ocasional $(17,5 \%$ y $13,0 \%$; $p<0,0001)$. Las prevalencias de fumadores explícitos fueron sistemáticamente mayores en mujeres en todos los NSE (Tablas 3 y 4), con la excepción de NSE bajo ( $43,2 \%$ en varones y $31,6 \%$ en mujeres).

Los escolares de NSE bajo presentaron la mayor prevalencia de tabaquismo explícito (36,8\%; 95\% IC: 35,5\%-38,2\%), contrastando con el NSE alto en el cual la prevalencia fue significativamente menor (31,6\%; 95\% IC: 30,2\%-32,9\%) (Tabla 2). En este NSE se observó además la mayor

\section{Tabla 2. Prevalencia de consumo de tabaco ${ }^{(*)}$ para diferentes grupos de edad, según sexo, nivel socioeconómico y zona geográfica de los alumnos entrevistados}

\begin{tabular}{|c|c|c|c|c|}
\hline \multirow[b]{2}{*}{ Variable } & \multicolumn{4}{|c|}{ Grupos de edad (años) $)^{(1)}$} \\
\hline & $\begin{array}{l}\text { 6-10 } \\
\mathrm{n} / \mathrm{N}(\%)\end{array}$ & $\begin{array}{l}11-14 \\
\mathrm{n} / \mathrm{N}(\%)\end{array}$ & $\begin{array}{l}\text { Más de } 14 \text { años } \\
\text { n/N (\%) }\end{array}$ & $\begin{array}{l}\text { Total } \\
\mathrm{n} / \mathrm{N}(\%)\end{array}$ \\
\hline \multicolumn{5}{|l|}{ Sexo } \\
\hline - Hombres & $4 / 435(0,9)$ & $345 / 2.643(13,1)$ & $1.832 / 4.366(42,0)$ & $2.181 / 7.444(29,3)$ \\
\hline - Mujeres & $2 / 428(0,5)$ & $390 / 2.602(15,0)$ & $2.426 / 4.645(52,2)$ & $2.818 / 7.675(36,7)$ \\
\hline \multicolumn{5}{|c|}{ Nivel socioeconómico(2) } \\
\hline - Alto & $0 / 283(0,0)$ & $163 / 1.744(9,4)$ & $1.435 / 3.036(47,3)$ & $1.598 / 5.063(31,6)$ \\
\hline - Medio & $3 / 283(1,1)$ & 240/1.754 $(13,7)$ & 1.324/3.037 (43,6) & $1.567 / 5.074(30,9)$ \\
\hline - Bajo & $3 / 297(1,0)$ & $332 / 1.747(19,0)$ & 1.499/2.938 $(51,0)$ & $1.834 / 4.982(36,8)$ \\
\hline \multicolumn{5}{|c|}{ Zona geográfica de vivienda ${ }^{(3)}$} \\
\hline - Norte & $4 / 294(1,4)$ & 232/1.746 (13,3) & $1.484 / 3.009(49,3)$ & $1.720 / 5.049(34,1)$ \\
\hline - Centro & $1 / 293(0,3)$ & $289 / 1.774(16,3)$ & $1.526 / 3.082(49,5)$ & $1.816 / 5.149(35,3)$ \\
\hline - Sur & $1 / 276(0,4)$ & $214 / 1.725(12,4)$ & $1.248 / 2.920(42,7)$ & $1.463 / 4.921(29,7)$ \\
\hline
\end{tabular}

${ }^{(1)}$ Grupos de edad equivalentes a: $1^{0}$ a $5^{0}$ año básico, $6^{0}$ a $8^{0}$ año básico y $1^{10}$ a $4^{0}$ año medio respectivamente. ${ }^{\left({ }^{2}\right)}$ Nivel socioeconómico estimado según dependencia del establecimiento: alto (establecimientos particulares pagados), medio (establecimientos particulares subvencionados), bajo (establecimientos fiscales). (3)Zona norte: $1^{\underline{a}}$ a $4^{\underline{a}}$ región; zona central: regiones $5^{\underline{a}}$, metropolitana y 6 a ; zona sur. regiones $7 \stackrel{a}{a}$ a $12 \underline{\text { ạ }}{ }^{(*)}$ Tabaquismo explícito: Tabaquismo diario más tabaquismo ocasional. 
Tabla 3. Prevalencia de tabaquismo y otras categorías relacionadas con consumo de tabaco según grupos de edad, nivel socioeconómico y zona geográfica de los alumnos entrevistados

\begin{tabular}{|c|c|c|c|c|}
\hline & \multicolumn{4}{|c|}{ Grupos de edad ${ }^{(1)}$} \\
\hline & $\begin{array}{l}\text { 6-10 años } \\
\mathrm{n}(\%)\end{array}$ & $\begin{array}{l}\text { 11-14 años } \\
\text { n (\%) }\end{array}$ & $\begin{array}{l}\text { Más de } 14 \text { años } \\
\text { n (\%) }\end{array}$ & $\begin{array}{l}\text { Total } \\
\text { n (\%) }\end{array}$ \\
\hline Fumador diario & $2(0,2)$ & $201(3,8)$ & $2.481(27,5)$ & $2.684(17,8)$ \\
\hline Fumador ocasional & $4(0,5)$ & $534(10,2)$ & $1.777(19,7)$ & $2.315(15,3)$ \\
\hline Ex fumador & $6(0,7)$ & $325(6,2)$ & $913(10,1)$ & $1.244(8,2)$ \\
\hline Sólo probó & $135(15,6)$ & $1.319(25,2)$ & $2.021(22,4)$ & $3.475(23,0)$ \\
\hline Nunca probó & $716(83,0)$ & $2.866(54,6)$ & $1.819(20,2)$ & $5.401(35,7)$ \\
\hline \multirow[t]{3}{*}{ Total } & $863(100)$ & $5.245(100)$ & $9.011(100)$ & $15.119(100)$ \\
\hline & \multicolumn{4}{|c|}{ Nivel socio económico $(2)$} \\
\hline & $\begin{array}{l}\text { Alto } \\
\text { n (\%) }\end{array}$ & $\begin{array}{l}\text { Medio } \\
\text { n (\%) }\end{array}$ & $\begin{array}{l}\text { Bajo } \\
\text { n (\%) }\end{array}$ & $\begin{array}{l}\text { Total } \\
\text { n (\%) }\end{array}$ \\
\hline Fumador diario & $866(17,1)$ & $695(13,7)$ & $1.123(22,5)$ & $2.684(17,8)$ \\
\hline Fumador ocasional & $732(14,5)$ & $872(17,2)$ & $711(14,3)$ & $2.315(15,3)$ \\
\hline Ex fumador & $475(9,4)$ & $465(9,2)$ & $304(6,1)$ & $1.244(8,2)$ \\
\hline Sólo probó & $1.101(21,7)$ & $1.197(23,6)$ & $1.177(23,6)$ & $3.475(23,0)$ \\
\hline Nunca probó & $1.889(37,3)$ & $1.845(33,3)$ & $1.667(33,5)$ & $5.401(35,7)$ \\
\hline \multirow[t]{4}{*}{ Total } & $5.063(100)$ & $5.074(100)$ & $4.982(100)$ & $15.119(100)$ \\
\hline & \multicolumn{4}{|c|}{ Zona geográfica de vivienda(3) } \\
\hline & Norte & Centro & Sur & Total \\
\hline & n $(\%)$ & n (\%) & n $(\%)$ & n $(\%)$ \\
\hline Fumador diario & $875(17,3)$ & $1.100(21,4)$ & $709(14,4)$ & $2.684(17,8)$ \\
\hline Fumador ocasional & $845(16,7)$ & $716(13,9)$ & $754(15,3)$ & $2.315(15,3)$ \\
\hline Ex fumador & $351(7,0)$ & $477(9,3)$ & $416(8,4)$ & $1.244(8,2)$ \\
\hline Sólo probó & $1.098(21,8)$ & $1.228(23,8)$ & $1.149(23,4)$ & $3.475(23,0)$ \\
\hline Nunca probó & $1.880(37,2)$ & $1.628(31,6)$ & $1.893(38,5)$ & $5.401(35,7)$ \\
\hline Total & $5.049(100)$ & $5.149(100)$ & $4.921(100)$ & 15.119 (100) \\
\hline
\end{tabular}

${ }^{(1)}$ Grupos de edad equivalentes a: $1^{\underline{0}}$ a $5^{0}$ año básico, $6^{0}$ a $8^{0}$ año básico y $1^{10}$ a $4^{\underline{0}}$ año medio respectivamente. ${ }^{(2)}$ Nivel socioeconómico estimado según dependencia del establecimiento: alto (establecimientos particulares pagados), medio (establecimientos particulares subvencionados), bajo (establecimientos fiscales). (3)Zona norte: $1^{\text {a }}$ a $4^{\text {a }}$ región; zona central: regiones $5 \underline{a}$, metropolitana y $6 \underline{a}$; zona sur: regiones $7 \stackrel{\text { a }}{\text { a }} 12^{2}$.

prevalencia de escolares que declararon no haber probado nunca cigarrillos (34,8\%; 95\% IC: 33,5\%36,0\%) (Tabla 3).

Según el sexo de los alumnos entrevistados, el tabaquismo explícito tuvo las mayores prevalencias en mujeres en todos los grupos de edad, niveles socioeconómicos y zonas geográficas del país (Tabla 4).

La edad promedio del primer contacto con el tabaco fue de 12,34 años (desviación estándar 1,96 años), siendo de 12,25 años (desviación estándar 2,1 años) en varones y de 12,4 años 
Tabla 4. Prevalencia de tabaquismo explícito ${ }^{(*)}$ para ambos sexo según grupos de edad, nivel socioeconómico y zona geográfica de vivienda de los alumnos entrevistados

\begin{tabular}{|llll|}
\hline & Varones & Mujeres & Valor $\mathrm{p}$ \\
\hline Grupos de edad $^{(1)}$ & & & \\
- - -10 & $4 / 435(0,9)$ & $2 / 428(0,5)$ & 0,021 \\
$\quad$ - $11-14$ & $345 / 2.643(13,1)$ & $390 / 2.602(15,0)$ & 0,047 \\
- 14 y más & $1.832 / 4.366(42,0)$ & $2.426 / 4.645(52,2)$ & $<10-4$ \\
Nivel socioeconómico(2) & & & \\
- Alto & & $921 / 2.462(37,4)$ & $<10-4$ \\
- Medio & $677 / / 2.601(26,0)$ & $1.006 / 2.985(33,7)$ & $<10-4$ \\
- Bajo & $561 / 2.089(26,9)$ & $891 / 2.228(40,0)$ & 0,00002 \\
& $943 / 2.754(34,2)$ & & \\
Zona geográfica de vivienda ${ }^{(3)}$ & & & $<10-4$ \\
- Norte & & $982 / 2.594(37,9)$ & $<10-4$ \\
- Centro & $738 / 2.455(30,1)$ & $1.016 / 2.530(40,2)$ & 0,0001 \\
\hline - Sur & $800 / 2.619(30,6)$ & $820 / 2.551(32,1)$ & \\
\hline
\end{tabular}

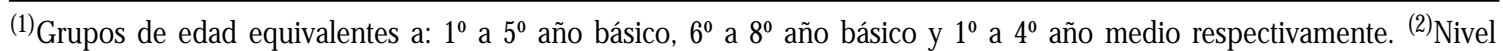
socioeconómico estimado según dependencia del establecimiento: alto (establecminetos particulares pagados), medio (establecimientos particulares subvencionados), bajo (establecimientos fiscales). ${ }^{(3)}$ Zona norte: $1^{\underline{a}}$ a $4^{\underline{a}}$ región; zona central:

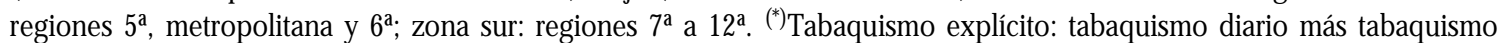
ocasional.

(desviación estándar 1,8 años). El promedio de edad declarado de fumar cigarrillos fue significativamente mayor que la edad de prueba de cigarrillos (13,16 años: desviación estándar 1,76 años). Los varones tuvieron menor edad promedio de inicio en la práctica de fumar $(13,10$ años; desviación estándar 1,88 años) que las mujeres (13,25 años; desviación estándar 1,66 años). Los escolares de NSE bajo tuvieron promedios de edad de prueba y de inicio en el fumar significativamente mayores que los otros estratos socioeconómicos. No hubo diferencias regionales en las variables de inicio del consumo.

Entre los fumadores explícitos, el $46 \%$ de ellos consumía menos de 5 cigarrillos entre lunes y viernes, porcentaje que disminuyó a $33,5 \%$ en fines de semana. No obstante, este cambio se acompañó de un aumento en el porcentaje de alumnos que consume más de 5 cigarrillos en fines de semana. En escolares con alto nivel de consumo (más de 20 cigarrillos entre lunes y viernes) éste también se incrementaba $(19,4 \%)$ en fines de semana.
Respecto del consumo a futuro, los fumadores explícitos declararon en alto porcentaje que mantendrán o aumentarán su actual nivel de consumo en los próximos cinco años (69,4\% de fumadores diarios y $55,5 \%$ en ocasionales). Contrasta con esta respuesta el deseo de mantenerse sin fumar en el futuro en los grupos de ex-fumadores, de quienes sólo probaron cigarrillos y de quienes nunca los probaron (71,8\%, 93,1\% y 97,4\% respectivamente).

El 15,4\% de fumadores explícitos no había pensado en dejar de fumar el año anterior y el $20 \%$ pensaba que podría dejar de fumar en cualquier momento. El 30\% de los fumadores explícitos pensaron en dejar de fumar, pero no habían hecho intentos para abandonar el hábito. Un porcentaje similar $(28,7 \%)$ señaló haber intentado dejar de fumar sin conseguirlo. El análisis por sexo no arrojó diferencias significativas en estas características.

Estimadores de riesgo de consumo de tabaco: las mujeres tuvieron $47 \%$ más de riesgo de probar cigarrillos o ser fumadoras explícitas que escolares varones, independiente de su NSE, región de residencia y edad (Tabla 5). Los escolares de NSE 
Tabla 5. Prevalencia de consumo de tabaco (tabaquismo explícito ${ }^{(*)}$ ) según variables seleccionadas. 0 dds de prevalencia crudos y ajustados ${ }^{(* *)}$

\begin{tabular}{|c|c|c|c|}
\hline Variable & n/N (\%) & $\begin{array}{l}\text { OR crudo } \\
\text { (95\% IC) }\end{array}$ & $\begin{array}{l}\text { OR ajustado } \\
\text { (95\% IC) }\end{array}$ \\
\hline \multicolumn{4}{|l|}{ Sexo } \\
\hline - Hombres & 2.181/7.444 (29,3) & 1 & 1 \\
\hline - Mujeres & 2.818/7.675 (36,7) & $1,4(1,31-1,50)$ & $1,47(1,36-1,6)$ \\
\hline \multicolumn{4}{|l|}{ Grupos Edad } \\
\hline - 6 a 10 & $6 / 863(0,7)$ & 1 & 1 \\
\hline-11 a 14 & $735 / 5.245(14,0)$ & $23,3(10,4-52,2)$ & $23,4(10,5-52,5)$ \\
\hline - Más de 14 & 4.258/9.011 (47,3) & $127,9(57,3-285)$ & $130(58,1-290,5)$ \\
\hline Nivel socioeconómico & $1.598 / 5.063(31,6)$ & 1 & 1 \\
\hline - Alto & $1.567 / 5.074(30,9)$ & $0,97(0,89-1,05)$ & $0,97(0,9-1,1)$ \\
\hline - Medio & $1.834 / 4.982(29,7)$ & $1,26(1,16-1,37)$ & $1,16(1,1-1,3)$ \\
\hline - Bajo & & & \\
\hline \multicolumn{4}{|l|}{ Zona } \\
\hline - Norte & $1.720 / 5.049(34,1)$ & 1 & 1 \\
\hline - Centro & $1.816 / 5.149(35,3)$ & $1,1(0,9-1,1)$ & $0,99(0,9-1,1)$ \\
\hline - Sur & $1.463 / 4.921(29,7)$ & $0,81(0,7-0,9)$ & $0,7(0,7-0,8)$ \\
\hline
\end{tabular}

${ }^{(*)}$ Tabaquismo explícito = Fumador ocasional y fumador diario. ${ }^{(*)}$ Cada variable ajustada recíprocamente por el resto de las otras variables de la tabla.

bajo presentaron mayor riesgo de tabaquismo explícito que los de NSE alto y medio (OR ajustado de 1,16; 95\% IC: 1,1-1,3). Los escolares de las regiones del sur tuvieron un menor riesgo de tabaquismo explícito que el resto de las zonas del país (OR ajustado 0,7: 95\% IC: 0,7-0,8). Los escolares de la región central fueron quienes presentaron un mayor riesgo de prueba o de consumo de cigarrillos durante su vida escolar en comparación con el resto de las regiones evaluadas (OR 1,4: IC 95\%: 1,2-1,5) (Tabla 6). No obstante observó el mayor riesgo de tabaquismo explícito en escolares mayores de 14 años, el cambio más significativo se observa en la transición entre niños de 6 a 10 años y los de 11 a 14 años de edad (OR de 13,9) (Tabla 5). El análisis del riesgo de probar o fumar cigarrillos según el curso de los escolares, señala una relación positiva y estadísticamente significativa en los cursos de enseñanza básica ( $p$ de tendencia menor a 0,0001).

Influencia de variables familiares y sociales del escolar; se estudió la relación entre hábito tabáquico de los padres y riesgo de tabaquismo explícito de sus hijos escolares (Tabla 7), constatándose un mayor riesgo de tabaquismo explícito si el padre o la madre tenían el hábito de fumar. Destacó especialmente la mayor influencia sobre el tabaquismo del niño cuando era la madre quien consumía tabaco (OR ajustado 1,9; IC: 1,7-2,0), riesgo mayor al observado cuando era el padre el consumidor (OR ajustado 1,5; IC: 1,4-1,6). El antecedente de tabaquismo paterno referido por los escolares fue de 47,6\% (49,9\% en escolares de NSE bajo) siendo la frecuencia reportada de tabaquismo materno de $46,3 \%$ (48,8\% en escolares de NSE alto). No se encontró evidencia de interacción entre tabaquismo materno y paterno respecto del riesgo de probar/fumar cigarrillos. Por otra parte, las existencia de reglas o restricciones parentales al consumo, se refleja en una reducción de $34 \%$ en la prevalencia de consumo explícito (OR 0,66; 95\% IC: 0,57-0,77) cuando los padres no dan autorización para fumar.

La provisión de cigarrillos por parte de los pares constituyó un importante factor de riesgo, triplicando el riesgo de tabaquismo, independiente del sexo y edad del entrevistado (Tabla 7). 
Tabla 6. Prevalencia y 0 dds de prevalencia crudos y ajustados de consumo de cigarrillos (probar/fumar) en escolares según sexo, nivel socioeconómico, zona geográfica de vivienda y curso (10 a 8ㅇ)

\begin{tabular}{|c|c|c|c|}
\hline Variable & n/N (\%) & $\begin{array}{l}\text { OR crudo } \\
\text { (95\% IC) }\end{array}$ & $\begin{array}{l}\text { OR ajustado }{ }^{1} \\
(95 \% \text { IC) }\end{array}$ \\
\hline \multicolumn{4}{|l|}{ Sexo } \\
\hline - Hombres & $4.474 / 7.408(60,4)$ & 1 & 1 \\
\hline - Mujeres & $5.172 / 7.639(67,7)$ & $1,4(1,29-1,50)$ & $1,4(1,3-1,50)$ \\
\hline \multicolumn{4}{|c|}{ Nivel socioeconómico } \\
\hline - Alto & $3.152 / 5.041(62,5)$ & 1 & 1 \\
\hline - Medio & $3.205 / 5.050(63,5)$ & $1,04(0,96-1,1)$ & $1,00(0,9-1,1)$ \\
\hline - Bajo & $3.289 / 4.956(66,4)$ & $1,2(1,1-1,3)$ & $1,3(1,1-1,4)$ \\
\hline \multicolumn{4}{|l|}{ Zona } \\
\hline - Norte & $3.143(62,6)$ & 1 & 1 \\
\hline - Centro & $3.499(68,3)$ & $1,3(1,2-1,4)$ & $1,4(1,2-1,5)$ \\
\hline - Sur & $3.004(61,3)$ & $0,9(0,9-1,04)$ & $0,9(0,8-1,02)$ \\
\hline \multicolumn{4}{|l|}{ Curso (básico) } \\
\hline-1 & 8/77 (10,4) & 1 & 1 \\
\hline-2 & $11 / 105(10,5)$ & $1,0(0,4-2,6)$ & $1,0(0,4-2,7)$ \\
\hline-3 & $34 / 155(21,9)$ & $2,4(1,1-5,5)$ & $2,5(1,09-5,7)$ \\
\hline-4 & $45 / 259(17,4)$ & $1,8(0,8-4,0)$ & $1,9(0,8-4,1)$ \\
\hline-5 & $48 / 266(18,0)$ & $1,9(0,9-4,2)$ & $1,9(0,8-4,1)$ \\
\hline-6 & $487 / 1.632(29,8)$ & $3,7(1,8-7,7)$ & $3,7(1,8-7,8)$ \\
\hline-7 & $775 / 1.677(46,2)$ & $7,4(3,5-15,5)$ & $7,7(3,7-16,1)$ \\
\hline-8 & $1.095 / 1.914(57,2)$ & $11,5(5,5-24,1)$ & $11,9(5,7-25,0)$ \\
\hline \multicolumn{4}{|c|}{$\begin{array}{l}p \text { tendencia } \\
p<10^{-4}\end{array}$} \\
\hline
\end{tabular}

${ }^{1}$ Odds de prevalencia ajustados por las variables comprendidas en la tabla.

Tabla 7. Prevalencia y 0 dds de prevalencia crudos y ajustados de consumo de cigarrillos (probar/fumar) en escolares según variables del entorno familiar y social

\begin{tabular}{|cll|}
\hline Variable & $\begin{array}{l}\text { OR crudo } \\
(95 \% \text { IC) }\end{array}$ & $\begin{array}{l}\text { OR ajustado } \\
(95 \% \text { IC) }\end{array}$ \\
\hline $\begin{array}{c}\text { Padres autorizan fumar } \\
-\quad \text { No }\end{array}$ & 1 & 1 \\
$-\quad$ Sí & $2,6(1,6-4,3)$ & $1,3(0,7-2,2)$ \\
Padre fumador & 1 & 1 \\
$-\quad$ No & $1,35(1,3-1,5)$ & $1,5(1,4-1,6)$ \\
$-\quad$ Sí & 1 & 1 \\
Madre fumadora & $1,8(1,6-1,9)$ & $1,9(1,7-2,0)$ \\
$-\quad$ No & 1 & 1 \\
- Sí & $5,3(4,8-5,7)$ & $3,2(3,0-3,6)$ \\
Amigos proveen cigarillos & No & \\
$-\quad$ Sí & & \\
\hline
\end{tabular}

${ }^{1}$ Ajustado por edad y sexo. 
Finalmente, el contexto de consumo más frecuente $(80 \%)$ para los escolares que fuman fue en las cercanías de los establecimientos educacionales, principalmente a la salida de los ellos o en plazas cercanas a él.

\section{DisCUSIÓN}

El estudio utilizó una muestra representativa de escolares en la que se cumplieron los supuestos utilizados en su cálculo, entre ellos, mayor prevalencia de consumo en mujeres, con la excepción de escolares menores de 11 años de edad. De los 46 establecimientos seleccionados, 13 de ellos decidieron no participar en la evaluación, siendo aleatoriamente substituidos en la muestra. A diferencia de otros estudios, se incluyó a escolares desde el $1^{\underline{0}}$ año básico en adelante, con la finalidad de poder estudiar la progresión del hábito tabáquico a lo largo de la vida escolar. El porcentaje de niños con edad superior a la esperada para su curso fue mayor en escolares de bajo NSE, lo que motivó un ajuste por edad de los niños en la elaboración de indicadores de riesgo del estudio.

El $64,1 \%$ de los escolares evaluados declaró haber tomado contacto directo con el cigarrillo durante algún momento de su vida. Esta experiencia de contacto con cigarrillos y de consumo habitual o esporádico fue significativamente mayor en mujeres, hecho que es independiente del NSE y zona geográfica de vivienda. Este fenómeno viene advirtiéndose sistemáticamente en los estudios nacionales y parece haberse instalado definitivamente en la población escolar ${ }^{8,32}$. Este comportamiento de las mujeres respecto del consumo de tabaco coincide con observaciones en otros países, sugiriendo una susceptibilidad particular al consumo en la población escolar femeni$\mathrm{na}^{28-31}$. Sin embargo, los varones tienen menor promedio de edad de inicio en el hábito, independiente del NSE y zona geográfica de los escolares estudiados.

La edad promedio de contacto con el cigarrillo, sea por prueba o iniciando su consumo regular, resulta estadísticamente menor en comparación con el promedio reportado en el estudio $\mathrm{CONACE}^{8}(\mathrm{t}=16,53, \mathrm{p}<0,0001)$.
Coincidiendo con los resultados obtenidos en otros estudios ${ }^{31}$, el riesgo de consumo habitual u ocasional de tabaco de adolescentes en presencia de una madre fumadora es mayor que el riesgo asociado al tabaquismo en presencia de consumo del padre. En la medida que la mujer adulta persista con la conducta tabáquica, en especial en el hogar, la prevención primaria del hábito será compleja y difícil de lograr por la fuerte influencia que ésta genera al interior de la familia. A partir de la estimación que hacen los hijos escolares, la prevalencia de tabaquismo paterno y materno reportada por los escolares es prácticamente la misma para ambos, revelando que los escolares no perciben diferencias por género en el hábito tabáquico de sus padres.

Al igual que la situación del adulto, y coincidiendo con información del Reino Unido ${ }^{30}$, los escolares de NSE bajo presentan la mayor prevalencia de contacto con el cigarrillo y de consumo ocasional o regular, lo que sugiere que el precio de los cigarrillos no constituye una barrera que impida el acceso a este producto, contrariamente a lo observado por Ivanovic ${ }^{14}$. Es posible que el precio explique en parte el mayor promedio de edad en el inicio del consumo observado en escolares de NSE bajo, limitación que parece no mantenerse una vez iniciado el hábito. El análisis de la progresión del contacto con el cigarrillo es relevante para la focalización de estrategias preventivas en el momento de mayor riesgo epidemiológico (Tabla 6). Respecto de la experiencia de prueba de cigarrillos, el momento crítico para la exposición corresponde a la transición entre $4^{\circ}$ y 5o año básico para los varones (9 a 10 años de edad) y para 50 y $6^{0}$ en las mujeres (10 a 11 años de edad). El consumo declarado de cigarrillos tiene su momento crítico entre $5^{\circ}$ y $6^{0}$ básico $(10 \mathrm{a}$ 11 años de edad) en niños y niñas. En consecuencia, parece ser que la mujer, una vez que entra en contacto con el cigarrillo, se encamina con mayor rapidez que el varón al consumo regular o esporádico, como lo refleja la mayor prevalencia de tabaquismo explícito en mujeres de enseñanza media. Los escolares que son ya fumadores habituales o esporádicos declaran globalmente su deseo de mantenerse fumando en cantidad igual o mayor que el consumo actual. Esta expectativa de consumo es mayor en mujeres que en varones. Complementariamente, el porcentaje de fumado- 
Tabla 8. Prevalencias reportadas por estudios previos sobre conducta tabáquica en escolares chilenos

\begin{tabular}{|c|c|c|c|c|c|c|c|}
\hline \multirow{2}{*}{$\begin{array}{l}\text { Autor } \\
\text { (Año) }\end{array}$} & \multirow{2}{*}{$\begin{array}{l}\text { Cursos } \\
\text { evaluado }\end{array}$} & \multicolumn{4}{|c|}{ Características evaluadas respecto a consumo de tabaco } & \multirow[t]{2}{*}{ Edad de Inicio } & \multirow{2}{*}{$\begin{array}{l}\text { Población } \\
\text { (n) }\end{array}$} \\
\hline & & Prueba & Fumador & Ex fumador & No fumador & & \\
\hline $\begin{array}{l}\text { Salas } \\
\text { (1979) }\end{array}$ & 3o Medio & - & $\begin{array}{l}58,3 \\
\text { Mujer } 59 \\
\text { Hombre 56,9 }\end{array}$ & 6,1 & 35,6 & 13-15 años & $\begin{array}{l}\text { Urbana } \\
\text { (312) }\end{array}$ \\
\hline $\begin{array}{l}\text { Vargas } \\
\text { (1980) }\end{array}$ & 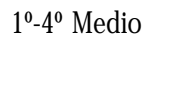 & - & $\begin{array}{l}55,6 \\
\text { Mujer 51,6 } \\
\text { Hombre 59,8 }\end{array}$ & & $\begin{array}{l}\text { Mujer } 48,4 \\
\text { Hombre } 40,2\end{array}$ & 13-14 años & $\begin{array}{l}\text { Urbana } \\
(565)\end{array}$ \\
\hline $\begin{array}{l}\text { Cabrera } \\
\text { (1982) }\end{array}$ & 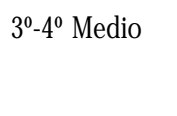 & - & $\begin{array}{l}56,5 \\
\text { Mujer } 53 \\
\text { Hombre 58,8 }\end{array}$ & - & $\begin{array}{l}44,5 \\
\text { Mujer } 47 \\
\text { Hombre } 41,2\end{array}$ & 13-15 años & $\begin{array}{l}\text { Urbana } \\
\text { (2172) }\end{array}$ \\
\hline $\begin{array}{l}\text { Ivanovic } \\
\text { (1986) }\end{array}$ & $\begin{array}{l}4^{0}-\text { - }^{0}-8^{0} \\
\text { Básico } \\
\text { 1ำ- Medio }^{0}\end{array}$ & - & $\begin{array}{l}\text { 4o Básico 0,5 } \\
\text { 6o Básico 6,1 } \\
\text { 8o Básico 11 } \\
\text { 10 Medio 22 } \\
\text { 4o Medio 36,7 }\end{array}$ & & (1) & - & $\begin{array}{l}\text { Urbano } \\
\text { (2967) }\end{array}$ \\
\hline $\begin{array}{l}\text { Sobarzo } \\
\text { (1987) }\end{array}$ & 10-4ํㅡㄴ Medio & - & $\begin{array}{l}\text { Hombre 36,7 } \\
\text { Mujer 17,4 }\end{array}$ & $\begin{array}{l}\text { Mujer 6,1 } \\
\text { Hombre } 4,9\end{array}$ & $\begin{array}{l}67,7 \\
\text { Mujer 76,5 } \\
\text { Hombre 58,4 }\end{array}$ & - & $\begin{array}{l}\text { Rural } \\
\text { (415) }\end{array}$ \\
\hline $\begin{array}{l}\text { Olivari } \\
\text { (1989) }\end{array}$ & 10-50 Básico & $\begin{array}{l}53,7 \\
\text { Mujer 47,6 } \\
\text { Hombre } 61\end{array}$ & $\begin{array}{l}40,7 \\
\text { Mujer 35 } \\
\text { Hombre 47,4 }\end{array}$ & & (1) & - & $\begin{array}{l}\text { Urbana } \\
\text { (1362) }\end{array}$ \\
\hline $\begin{array}{l}\text { Daszenies } \\
\text { (1999) }\end{array}$ & 1-4⿳⺈ Medio & $\begin{array}{l}\text { Mujer 57,7 } \\
\text { Hombre } 63,9\end{array}$ & $\begin{array}{l}\text { Mujer } 13,7 \\
\text { Hombre } 10,4\end{array}$ & - & - & - & $\begin{array}{l}\text { Urbana/Rural } \\
\text { (312) }\end{array}$ \\
\hline $\begin{array}{l}\text { CONACE } \\
\text { (2001) }\end{array}$ & 8-4-4 Medio & - & $\begin{array}{l}\text { Mujer 79,6\% } \\
\text { Hombre72,5\% }\end{array}$ & & & & $\begin{array}{l}58.722 \\
86 \text { comunas } \\
\text { urbanas }\end{array}$ \\
\hline $\begin{array}{l}\text { OMS Estudio } \\
\text { Intemacional) }\end{array}$ & $\begin{array}{l}\text { 80 Básico - 1ํ } \\
\text { Medio }\end{array}$ & 69,6 & 39,6 & - & 30,4 & - & $\begin{array}{l}\text { Coquimbo } \\
(1.746)\end{array}$ \\
\hline (2000) & (13-14 años) & 71,5 & 38,4 & - & 28,5 & - & $\begin{array}{l}\text { Santiago } \\
(3.150)\end{array}$ \\
\hline & & 68,4 & 36,1 & - & 31,6 & - & $\begin{array}{l}\text { Valparaíso } \\
\text { (1.452) }\end{array}$ \\
\hline $\begin{array}{l}\text { U.Católica } \\
\text { (2001) }\end{array}$ & $\begin{array}{l}1^{0} \text { Básico- } 4^{0} \\
\text { E Media }\end{array}$ & 64,3 & $\begin{array}{l}\text { Mujeres 36,7 } \\
\text { Hombres 29,8 }\end{array}$ & 8,2 & 35,7 & 12,4 años & $\begin{array}{l}\text { Muestra } \\
\text { Regional }\end{array}$ \\
\hline
\end{tabular}

res con expectativas de abandonar el cigarrillo en los próximos 5 años es menor en mujeres que en varones. La cesación del hábito parece no constituir una preocupación para los escolares fumadores.

En síntesis, constatamos elevada prevalencia de contacto con el cigarrillo y tabaquismo explícito en la muestra evaluada. La información obtenida en esta investigación, complementa la información existente, sugiriendo que el riesgo de consumo se produce precozmente en la vida escolar, con edades cada vez más tempranas en la iniciación del hábito. Es preocupante la situación de la mujer, ya que la prevalencia del consumo es mayor en la mujer desde temprana edad y puede estar en el contexto de un cambio asociado al género respecto de la percepción de este hábito en la mujer. Este fenómeno debe ser estudiado en forma específica, a fin de establecer estrategias preventivas ad hoc. El estudio de las características del grupo de escolares 
que en forma espontánea se mantienen al margen del consumo de tabaco ofrece una alternativa interesante de estudio.

A la luz de estos resultados, las políticas de control y prevención del hábito en la población escolar deben iniciarse precozmente en la vida, adelantándose a los momentos críticos para el consumo identificado en el análisis de la progresión del hábito según curso al cual asiste el escolar.

\section{REFERENCIAS}

1. Albala C, Vio F, Yáñez M. Transición epidemiológica en América Latina: comparación de cuatro países. Rev Méd Chile 1997; 125: 719-27.

2. Berríos $\mathrm{X}$, Jadue L, Zenteno J, Ross MI, Rodríguez $\mathrm{H}$. Prevalencia de factores de riesgo de enfermedades crónicas. Estudio en población general de la región metropolitana. Rev Méd Chile 1990; 118: 597-604.

3. Jadue L, Vega J, Vega M, Escobar MC, Garrido C, Lastra P ET al. Factores de riesgo para las enfermedades no transmisibles: metodología y resultados globales de la encuesta de base del programa CARMEN. Rev Méd Chile 1999; 127: 1004-13.

4. Medina E, KaempFFer AM. Health damage caused by the smoking habit in Chile. Bull Pan Am Health Organ 1991; 25: 248-57.

5. Lanas F, Davis MR, Standen D, Iuesca M, Doghetti $\mathrm{J}$, Sтоскіns B. Prevalencia de factores de riesgo cardiovascular en la ciudad de Temuco. Rev Méd Chile 1991; 119: 247-51.

6. Gutiérrez M, Rioseco F, Rojas A, Casanova D, Cordero M, Schiaffino M. Prevalencia de tabaquismo en población general de Valparaíso y Viña del Mar. Rev Méd Chile 1995; 123: 250-6.

7. Medina E, Kaempfrer AM, Cornejo E, Hernández E. Tabaquismo en Santiago, 1993-94. Rev Méd Chile 1995; 123: 652-8.

8. Consejo Nacional para el Control de Estupefacientes (CONACE). Ministerio del Interior. Gobierno de Chile. Cuarto estudio nacional sobre el consumo de drogas en población general de Chile 2000. Santiago de Chile, Mayo 2001.
Estrategias combinadas, con participación del núcleo familiar, del sistema escolar y del componente comunitario, son de urgente desarrollo. Estas deben contar con el apoyo de disciplinas científicas provenientes de las ciencias sociales, las que deberán proveer de marcos conceptuales capaces de reforzar las capacidades individuales de los escolares para mantenerse en abstinencia tabáquica.

9. Vargas na, Carretero A, Salomon V, Ramírez B, CouRBIS E. Hábito tabáquico en población escolar urbana. Rev Chil Pediatr 1980; 51: 379-85.

10. Cabrera F, Salomon C, López I, Vidal R. Hábito tabáquico de estudiantes secundarios en Santiago, Chile. Bol Oficina Sanit Panam 1982; 93: 53340.

11. Salas I, Ramos E, Peters G, Pesenti L, O’Ryan F, NIEme E et al. Prevalencia de tabaquismo en tercer año de enseñanza secundaria. Rev Méd Chile 1982; 110: 1237-44.

12. Sobarzo P, Muñoz J, Vargas M, Behn V. Tabaquismo en estudiantes de enseñanza media de una comunidad rural. Rev Méd Chile 1988; 116: 82935.

13. Olivari F, De la Fuente M, López I. Hábito de fumar en escolares de enseñanza básica. Estudio en una población de bajo nivel socioeconómico. Rev Méd Chile 1989; 117: 861-6.

14. Ivanovic, D, Castro C, Ivanovic R. Factores que inciden en el hábito de fumar de escolares de educación básica y media de Chile. Rev de Saúde Pública 1997; 131: 30-43.

15. Alvarado R, Román E, Sayago S, Soto I, Pinto R, Horta N. Consumo de tabaco entre estudiantes de enseñanza media. I Descripción del Hábito Tabáquico y de Factores Asociados. Cuad Méd Soc 1998; XXXIX: 56-70.

16. Daszenies C, Covacevich C. Tabaquismo en estudiantes de enseñanza media en una comunidad rural de Chiloé. Rev Méd Chile 1999; 127: 38-43.

17. The Global Youth Tobacco Survey Colmaborative Group. Tobacco use among youngs: a cross country comparision. Tobacco Control 2002; 11: 252-70. 
18. Consejo Nacional para el Control de Estupefacientes (CONACE). Ministerio del Interior. Gobierno de Chile. Estudio Nacional de drogas en la población escolar de Chile, 2001. De 8o Básico a 4º Medio. Santiago de Chile, Mayo 2002.

19. Biglan A, Duncan T, Ary DV, Smolkowski K. Peer and parental influences on adolescent tobacco use. Journal of Behavioral Medicine 1995; 18: 315-30.

20. A model for change: The California experience in tobacco control. California Dept. Health Services/ Tobacco Control Section, Sacramento, October, 1998 California, USA.

21. Chassin L, Presson CC, Rose JS, Sherman SJ. Maternal socialization of adolescent smoking: The intergenerational transmission of parenting and smoking. Dev Psychology 1998; 34: 1189-201.

22. Alvarado R, Román E, Sayago S, Soto I, Pinto R, Horta N. Consumo de tabaco entre estudiantes de enseñanza media. II Construcción de un Modelo Explicativo para su consumo. Cuad Méd Soc 1998; XXXIX: 71-82.

23. Chen X, Unger JB, Palmer P, Weiner MW, Johnson CA, Wong MM et aL. Prior cigarette smoking initiation predicting current alcohol use: Evidence for the gateway drug effect among California adolescents from eleven ethnic groups. Addictive Behaviors 2002; 27: 799-817.

24. Health Behaviour in School-aged Children. A WHO Cross-National Survey (HBSC). Research protocol for the 1997-98 study. Dept. of Community Health ciences, University of Edimbugh.

25. DOHeRTy W, Auen W. Family functioning and parental smoking as predictors of adolescent cigarette use: a six years prospective study. Journal of Family Psychology 1994; 8: 347-53.
26. North Caroline Youth Tobacco Survey (YTS) 1999 Questionnaire. Division of Public Health, North Carolina Department of Health and Human Services.

27. Stacy Alan, Flay B, Sussman S, Brown S, Santi S, BEST A. Validity of alternative self-report indices of smoking among adolescents. Psychological Assement 1990; 2: 442-6.

28. Molarius A, Parsons RW, Dobson AJ, Evans A, Fortmann SP, Jamrozik K, KuUlasmaa K, Moltchanov $\mathrm{V}$, Sans S, Tuomilehto J, Puska P, WHO MONICA Project. Trends in cigarette smoking in 36 populations from the early 1980s to the mid 1990s: findings from the WHO MONICA Project. Am J Public Health 2001; 91: 206-12.

29. Wechsler H, Rigotti N, GleghiL-Hoyt J, Lee $\mathrm{H}$. Increased levels of cigarette use among college students: A cause for national concern. JAMA 1998; 280: 1673-8.

30. Molyneux A, Lewis S, Antoniak M, Hebbard R, McNeill A, Godfrey C et AL. Is smoking a communicable disease? Effect of exposure to ever smokers in school tutor groups on the risk of incident smoking in the first year of secondary school. Tobacco Control 2002; 11: 241-5.

31. Morelo P, Duggan A, Adger H, Anthony J, Joffe A. Tobacco use among high school students in Buenos Aires, Argentina. Am J Pub Health 2001; 91: 219-24.

32. Informe Final Encuesta Mundial de Tabaquismo en Jóvenes (EMTAJOVEN), Santiago de Chile, septiembre de 2001. Universidad de Chile, Facultad de Medicina, Escuela de Salud Pública. www.cdc.gov/tobacco/global/GYTS/ reports/ chile2001 sp.htm 